\section{Recovery from pneumonia requires efferocytosis which is impaired in smokers and those with low body mass index and enhanced by statins}

\begin{abstract}
Background Efferocytosis (the phagocytosis of apoptotic self cells) is a key mechanism in the resolution of inflammatory processes such as community-acquired pneumonia (CAP).

Efferocytosis therefore represents a modifiable target for therapy aimed at enhancing intrinsic recovery mechanisms. It is currently not known which patients recovering from CAP would mostly benefit from a strategy aimed at enhancing efferocytosis.
\end{abstract}

Methods We recruited a cohort of patients with CAP admitted to a hospital in Liverpool. One month into recovery, subjects were invited for research bronchoscopy and bronchoalveolar lavage. An ex vivo efferocytosis assay was performed by challenging alveolar macrophages with autologous, apoptotic neutrophils. The percentage of alveolar macrophages that had undergone efferocytosis was determined by flow cytometry. We conducted a multivariable regression using a linear mixed effects model to determine which clinical parameters were most closely associated with efferocytosis.

Results We observed high rates of comorbidity among this CAP cohort. Efferocytosis was measured in 22 subjects. We assessed multiple combinations of clinical parameters for association with efferocytosis and found the best-fitting model included an interaction between smoking status and prior statin use-smoking being associated with decreased efferocytosis and statin use with increased efferocytosis. These effects were modified by an association between efferocytosis and body mass index (BMI), such that as BMI increased so did efferocytosis. Conclusions This is the first study to measure efferocytosis in patients recovering from CAP. The results suggest that smokers with low BMI have impaired efferocytosis and may benefit from a statin to boost recovery.

\section{INTRODUCTION}

Among the $80 \%$ of patients who survive an admission with community-acquired pneumonia (CAP), a proportion suffers prolonged symptoms. ${ }^{1}$ During recovery from pneumonia, dead and dying neutrophils must be cleared. This phagocytosis of apoptotic 'self' cells, called efferocytosis, is defective in idiopathic pulmonary fibrosis and COPD. ${ }^{2}$ The efferocytosis of apoptotic neutrophils has been studied in pneumonic mice but not in humans recovering from $\mathrm{CAP}^{3}$ In patients with CAP, clinical severity markers are associated with short-term mortality risk, but the association of these factors with efferocytosis following CAP is not known. If pre-CAP clinical factors were associated with efferocytosis, then pro-resolution therapy to reduce post-CAP adverse events would become a possibility. We conducted a prospective cohort study of adults hospitalised for CAP. We hypothesised that ex vivo efferocytosis of autologous apoptotic neutrophils by alveolar macrophages would vary in association with patient characteristics.

\section{METHODS}

Subjects recruited from two UK Hospitals between February 2011 and March 2013 had CAP (British Thoracic Society definition), were aged $>16$ years and were recruited within 24 hours of their first dose of in-hospital antibiotic. We excluded patients requiring invasive ventilation, requiring renal replacement therapy, with cystic fibrosis (CF), non-CF bronchiectasis, lung cancer, lung metastases, advanced cancer of any type, immunocompromise (including systemic corticosteroids), those treated palliatively or admitted within 14 days. At 1 month, subjects were invited for bronchoscopy with bronchoalveolar lavage (BAL) and were excluded if they were at increased risk of complications. BAL was performed as previously published with $200 \mathrm{ml}$ saline instilled into the right middle lobe (RML) bronchus. ${ }^{4}$ BAL was filtered, pelleted, washed and resuspended in Iscove's Modified Dubecco's Medium (IMDM) with human $\mathrm{AB}$ serum and antibiotics. Cells were seeded into 48 well plates and incubated for 4 hours before the medium was replaced with antibiotic-free IMDM.

Ex vivo autologous apoptotic neutrophils were derived by published protocol. ${ }^{5}$ Prior to incubation, the neutrophils were divided into two aliquots: one stained green and the other unstained. The efferocytosis assay was a modification of published protocols. $^{6}$ After overnight incubation, the media was removed from the macrophages and unstained neutrophils, stained neutrophils or medium added to each well (see online supplementary figure S1). After $90 \mathrm{~min}$ co-culture, the medium was removed and the macrophages washed to remove uningested neutrophils. Macrophages were detached with cold phosphate-buffered saline (PBS), washed, quenched and then acquired on the cytometer.

The flow cytometric gating strategy (see online supplementary figure S2) involved the separation of uningested neutrophils from macrophages by light scatter as previously published. ${ }^{6}$ Using $\mathrm{R}$, associations with efferocytosis were analysed by multiple regression. The mean of efferocytosis experimental replicates was used for univariate analysis; then, a maximal linear mixed effects model was constructed using efferocytosis as the response variable, correlated $(p>0.05)$ variables from the univariate analysis as fixed effects and 'subject id' as a random effect. The random effect enabled us to separate the stochastic variation associated with experimental technique from between subject variations. Backwards elimination derived the minimum set of explanatory variables required to give a statistically acceptable fit.

\section{RESULTS}

Of 169 subjects recruited, efferocytosis was analysed in 22 (figure 1 and online supplementary tables S1 and S2). Univariate analysis (see online supplementary table S3) revealed a trend towards higher efferocytosis values with improved symptomatic recovery (see online supplementary figure S3), but no relationship with age. Smoking status, prior statin use, body mass index (BMI) and gender correlated with efferocytosis. Those variables were combined in a linear mixed effects model which left three variables with statistically significant effects: smoking status, prior statin use and BMI. As BMI increased, so did efferocytosis. Smoking was associated with lower rates of efferocytosis. Subjects who were taking statins had higher rates of efferocytosis. Analysis for interactions showed the model with the best data-fit included an interaction between smoking status and prior statin use, with adjustment for BMI (figure 2). The interaction was such that the statin-associated increase in efferocytosis was largest in those who were active smokers. The final model explained $42.6 \%$ of variation in the data, of which $90.1 \%$ was the difference between patients and 9.9\% was within experimental replicates.

\section{DISCUSSION}

This is the first study of efferocytosis during recovery from CAP. After adjustment for BMI, the strongest associations with efferocytosis were smoking status and statin use prior to CAP.

Strengths of this study include the use of autologous neutrophils as a pathophysiologically appropriate apoptotic target for the efferocytosis assay, the use of linear mixed effects modelling to quantify the contributions of experimental and between-patient variations and the flow cytometric method used. Limitations 


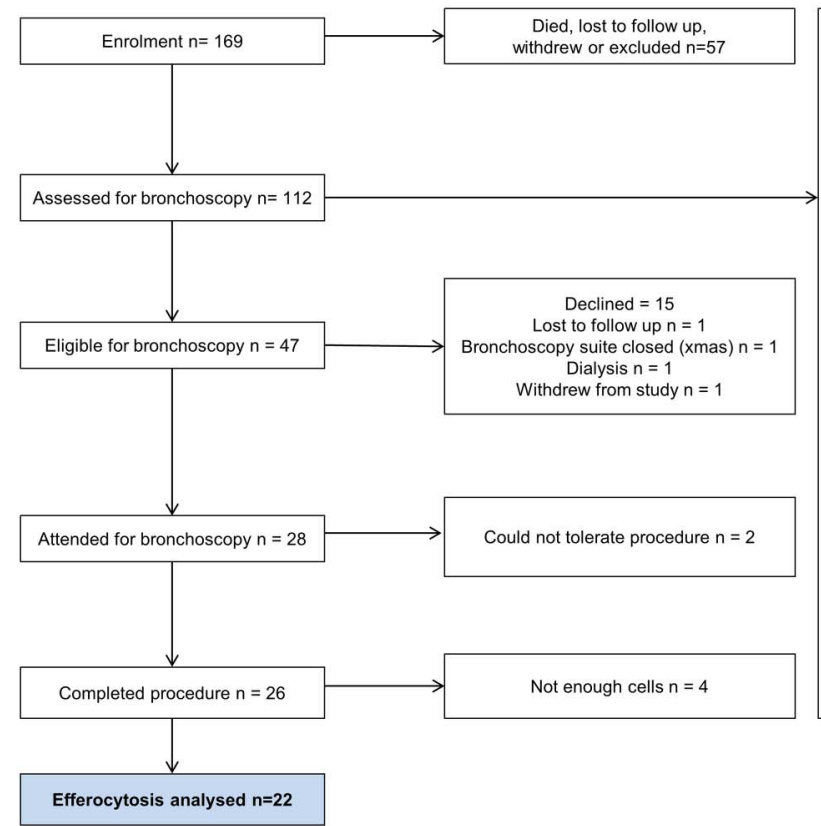

In jail $\mathrm{n}=1$

Not eligible due to comorbidity:-

Severe COPD $n=19$

$\mathrm{AF}$ and on warfarin $n=13$

Frailty $n=4$

Recent clinical bronchoscopy $n=3$

Myocardial infarction within 6 weeks $n=1$

Unresolved severe CAP $=2$

Hypoxia at rest $n=2$

Congestive cardiac failure $n=2$

Aortic valve replacement on warfarin $n=1$

Mitral valve replacement on warfarin $n=1$

Severe aortic stenosis $n=1$

Advanced multiple sclerosis $n=1$

Latex allergy $n=1$

Pregnant $\mathrm{n}=1$

Brittle asthma $n=1$

Brittle diabetes $n=1$

Severe obstructive sleep apnoea $n=1$

Severe parkinsons $n=1$

Multiple rib fractures $n=1$

Diaphragmatic paralysis $n=1$

Laryngectomy $n=1$

Un-clipped cerebral aneurysm $n=1$

Severe thrombocytopaenia (ITP) $n=1$

Severe vocal cord dysfunction $n=1$

Dilated cardiac myopathy $n=1$

Hemiparesis $=$

Total not eligible $n=65$

Figure 1 Bronchoscopy flow chart. Approximately half of the subjects assessed were deemed ineligible for research bronchoscopy-the majority of those being excluded on safety grounds due to comorbidity. CAP, community-acquired pneumonia

include the small study size, lower median age and lower range of severity than that in clinical practice. It is not known if CAP in humans has an effect on efferocytosis or whether any such effect would be local or generalised, but we consistently lavaged the RML, and three of the patients were recovering from RML CAP. It is possible that recent local inflammation by the RML CAP may have affected rates of local efferocytosis; however, in our univariate analysis, there was no statistically significant difference in efferocytosis associated with RML involvement.
Previous studies have found that cigarette smoke affects molecular pathways that lead to the activation and membrane localisation of the enzyme Rac which facilitates the cytoskeletal rearrangements needed for efferocytosis. ${ }^{7}$ Statins cause the enzymes Rac and RhoA to sequester

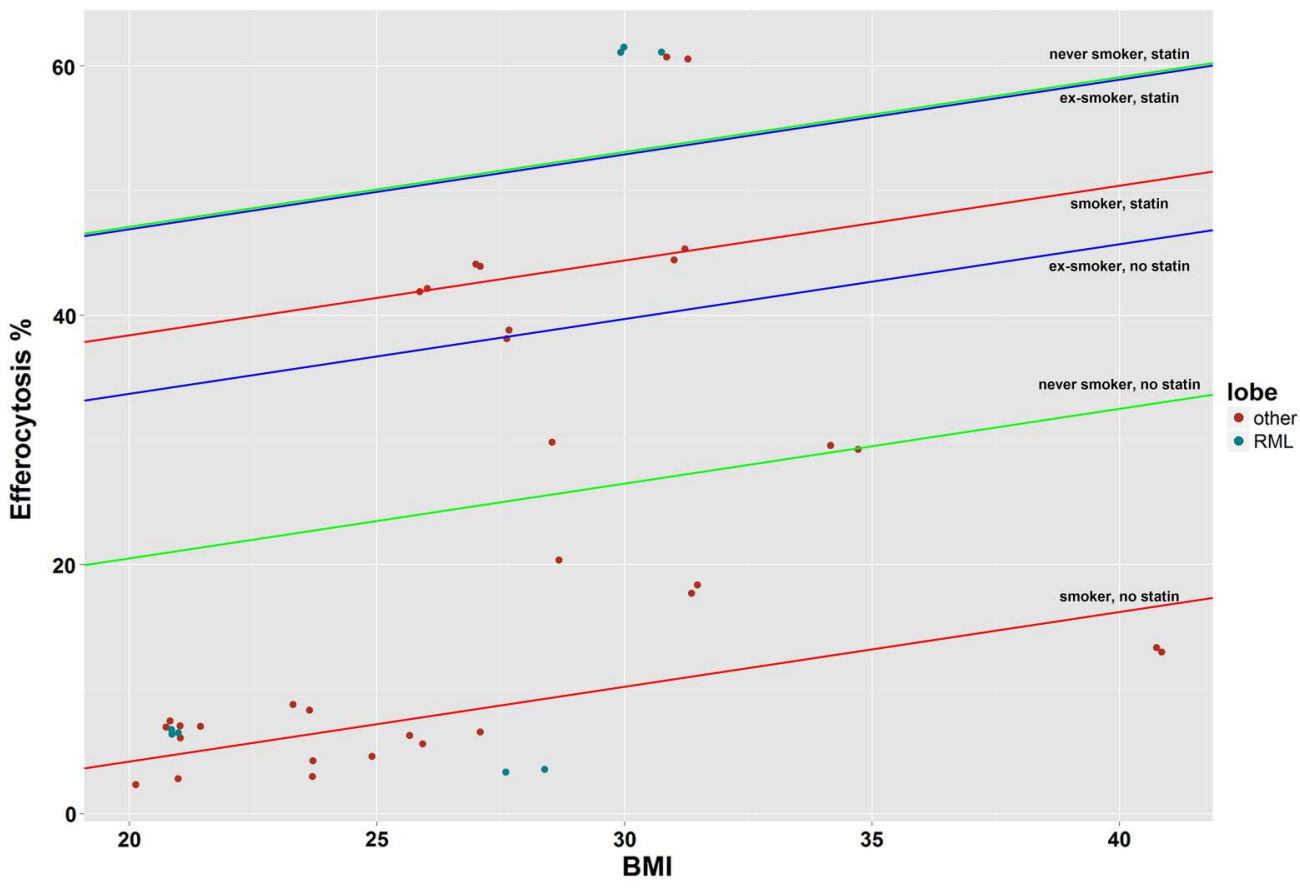

Figure 2 Interactions between covariates in the linear mixed effects model. The result of the linear regression was a model that included the interaction of smoking and statin with adjustment for body mass index (BMI). The efferocytosis values fitted by the model (including all replicates) are on the $\mathrm{Y}$-axis, with BMI on the X-axis. Points are 'jittered' to avoid overlap. The modelled interactions of each level of smoking and statin are represented by six straight lines. These lines are displayed in colour pairs based on smoking status. The y-intercepts of each line are derived from the model, as is the BMI-dependent slope of 0.6. From these lines, it can be seen that without statins the differences between smoking status are large, but with statins the differences are smaller. The magnitude of the statin effect appears greatest in smokers. There was no association between efferocytosis and sampling from the right middle lobe (RML). 
in the cytosol, resulting in increased efferocytosis of apoptotic neutrophils. ${ }^{8}$ These studies suggest smoking and statins have antagonistic effects on Rac1 and RhoA, and as a consequence opposite effects on efferocytosis, providing a possible mechanistic explanation for our finding of a negative association between efferocytosis and smoking, a positive association with statins and a statistical interaction between smoking and statin use.

We also showed a novel association between BMI and efferocytosis. Previous studies have shown that reduced CAP mortality is associated with high $\mathrm{BMI}^{9}$ and that low BMI is associated with increased risk of developing $\mathrm{CAP}^{10}$ Differential rates of efferocytosis may explain these correlations.

Our study suggests that smokers with CAP and low BMI may benefit most from augmented efferocytosis; a statin would be an appropriate candidate for such a trial.

\section{Daniel G Wootton, ${ }^{1,2}$ Peter J Diggle, ${ }^{3}$ Joanne Court, ${ }^{4}$ Odiri Eneje, ${ }^{4}$ Lynne Keogan, ${ }^{2}$ Laura Macfarlane, ${ }^{4}$ Sarah Wilks, ${ }^{4}$ Mark Woodhead, ${ }^{5,6}$ Stephen B Gordon ${ }^{3}$}

${ }^{1}$ Institute of Infection and Global Health, University of Liverpool, Liverpool, UK

${ }^{2}$ Department of Respiratory Research, Aintree University Hospital NHS Foundation Trust, Liverpool, UK ${ }^{3}$ CHICAS, Lancaster University Medical School, Lancaster University, Lancaster, UK

${ }^{4}$ Department of Clinical Sciences, Liverpool School of Tropical Medicine, Liverpool, UK

${ }^{5}$ Department of Respiratory Medicine, Central Manchester University Hospitals NHS Foundation Trust, Manchester, UK

${ }^{6}$ Manchester Academic Health Science Centre and Faculty of Medical and Human Sciences, University of Manchester, Manchester, UK

Correspondence to Dr Daniel G Wootton, Department of Clinical Infection, Microbiology and Immunology, Institute of Infection and Global Health, University of Liverpool, 8 West Derby Street, Liverpool L69 7BE, UK; dwootton@liverpool.ac.uk

Acknowledgements The authors thank the patients who volunteered for the study, the NIHR for funding the work and the NIHR Clinical Local Research Network (CLRN) for providing strategic support for recruitment.

Contributors DGW conceived the study, wrote the grant to obtain the funding, defended the ethics, participated in recruitment, followed up the subjects, performed all bronchoscopies, performed all lab assays, analysed the results and wrote the manuscript. JC, LK, LM and SW helped recruit the patients, collate the data and reviewed the manuscript. In addition LK assisted with the BALs, recovery post bronchoscopy and patient follow-up. PJD and SBG helped design the study, write the grant, and along with MW supervised the fellowship project including discussion of recruitment, data analysis and the writing of this manuscript. PJD supervised the statistical plan and analysis.

Funding The study was funded by the National Institute of Health Research (NIHR) Doctoral Research Fellowship awarded to DG Wootton with support from the North West CLRN.

Competing interests None declared.
Ethics approval This work was approved by the UK NHS Research Ethics Committee (NHS Research Ethics Committee (REC) Number 10/WNo03/40), was sponsored by Aintree University Hospital NHS Foundation Trust and was listed on the NIHR Clinical Research Network portfolio. All subjects provided informed consent to join the study. A consultee provided assent on behalf of those who lacked capacity as a consequence of CAP-related delirium - with consent being retrospectively obtained upon recovery of capacity. In addition, at follow-up, all volunteers provided informed consent for bronchoscopy and BAL.

Provenance and peer review Not commissioned; externally peer reviewed.

- Additional material is published online only. To view please visit the journal online (http://dx.doi.org/ 10.1136/thoraxjnl-2016-208505).

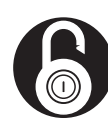

\section{OPEN ACCESS}

Open Access This is an Open Access article distributed in accordance with the terms of the Creative Commons Attribution (CC BY 4.0) license, which permits others to distribute, remix, adapt and build upon this work, for commercial use, provided the original work is properly cited. See: http:/l creativecommons.org/licenses/by/4.0/

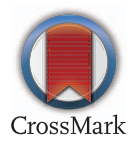

To cite Wootton DG, Diggle PJ, Court J, et al. Thorax 2016;71:1052-1054.

Received 19 February 2016

Revised 31 May 2016

Accepted 12 June 2016

Published Online First 28 July 2016

Thorax 2016;71:1052-1054.

doi:10.1136/thoraxjn-2016-208505

\section{REFERENCES}

1 El Moussaoui R, Opmeer BC, de Borgie CA, et al. Long-term symptom recovery and health-related quality of life in patients with mild-to-moderate-severe community-acquired pneumonia. Chest 2006;130(Suppl 4):1165-72.

2 McCubbrey AL, Curtis JL. Efferocytosis and lung disease. Chest 2013;143(Suppl 6):1750-7.

3 Morimoto K, Amano H, Sonoda F, et al. Alveolar macrophages that phagocytose apoptotic neutrophils produce hepatocyte growth factor during bacterial pneumonia in mice. Am I Respir Cell Mol Biol 2001;24(Suppl 5):608-15.

4 Collins AM, Rylance J, Wootton DG, et al. Bronchoalveolar lavage (BAL) for research; obtaining adequate sample yield. J Vis Exp 2014;(85):e4345. doi: $10.3791 / 4345$

5 Taylor EL, Rossi AG, Dransfield I, et al. Analysis of neutrophil apoptosis. Methods Mol Biol 2007:412:177-200.

6 Michlewska S, Dransfield I, Megson IL, et al. Macrophage phagocytosis of apoptotic neutrophils is critically regulated by the opposing actions of pro-inflammatory and anti-inflammatory agents: key role for TNF- $\alpha$. FASEB J 2009;23 (Suppl 3):844-54.

7 Noda N, Matsumoto K, Fukuyama S, et al. Cigarette smoke impairs phagocytosis of apoptotic neutrophils by alveolar macrophages via inhibition of the histone
deacetylase/Rac/CD9 pathways. Int Immunol 2013;25 (Suppl 11):643-50.

8 Morimoto K, Vandivier RW, Janssen WJ, et al. Lovastatin enhances clearance of apoptotic cells (efferocytosis) with implications for chronic obstructive pulmonary disease. J Immunol 2006;176 (Suppl 12):7657-65.

9 Kahlon S, Eurich DT, Padwal RS, et al. Obesity and outcomes in patients hospitalized with pneumonia. Clin Microbiol Infect 2013;19 (Suppl 8):709-16.

10 Phung DT, Wang Z, Rutherford S, et al. Body mass index and risk of pneumonia: a systematic review and meta-analysis. Obes Rev 2013;14 (Suppl 10):839-57. 